\title{
On Point Sets with Many Unit Distances in Few Directions
}

\author{
P. Brass \\ Institut für Informatik, FU Berlin, \\ Takustrasse 9, D-14195 Berlin, Germany \\ brass@inf.fu-berlin.de
}

Communicated by János Pach

\begin{abstract}
We study the problem of the maximum number of unit distances among $n$ points in the plane, under the additional restriction that we count only those unit distances that occur in a fixed set of $k$ directions, taking the maximum over all sets of $n$ points and all sets of $k$ directions. We prove that, for fixed $k$ and sufficiently large $n>n_{0}(k)$, the extremal sets are essentially sections of lattices, bounded by edges parallel to the $k$ directions and of equal length.
\end{abstract}

\section{Introduction}

The problem of the maximum number of unit distances is perhaps the simplest-sounding of Erdős's many combinatorial geometry problems; nonetheless it turned out to be a very difficult problem which is still far from solution. The question "How often can the same distance appear among $n$ points in the plane?" was first studied by Erdôs in his 1946 paper [9], where he constructed an upper bound of order $O\left(n^{3 / 2}\right)$ and a lower bound of order $\Omega\left(n e^{c(\log n / \log \log n)}\right)$, the last by taking a square section of a properly scaled integer lattice. The upper bound was then reduced in several steps [1], [12] to $O\left(n^{4 / 3}\right)$ [16]. Since the first proof was very complicated, alternative proofs were sought ([5] by "random resampling," [13] by use of the VC-dimension) until 1996 Székely found a very elegant proof using a crossing-number argument [17].

There has been no change in the lower bound: although triangular lattice sections seem to give slightly better numbers than square lattice sections, they have the same asymptotics, and lattice sections are still the best-known construction. Erdős repeatedly offered $\$ 250$ for an upper bound which is $O\left(n^{1+\varepsilon}\right)$ for all $\varepsilon>0$ and $\$ 500$ for an upper bound $O\left(n e^{c(\log n / \log \log n)}\right)$. Exact values and extremal sets for up to 14 points (Fig. 1) were determined by Schade [15] (see also [2]). Also many related problems 


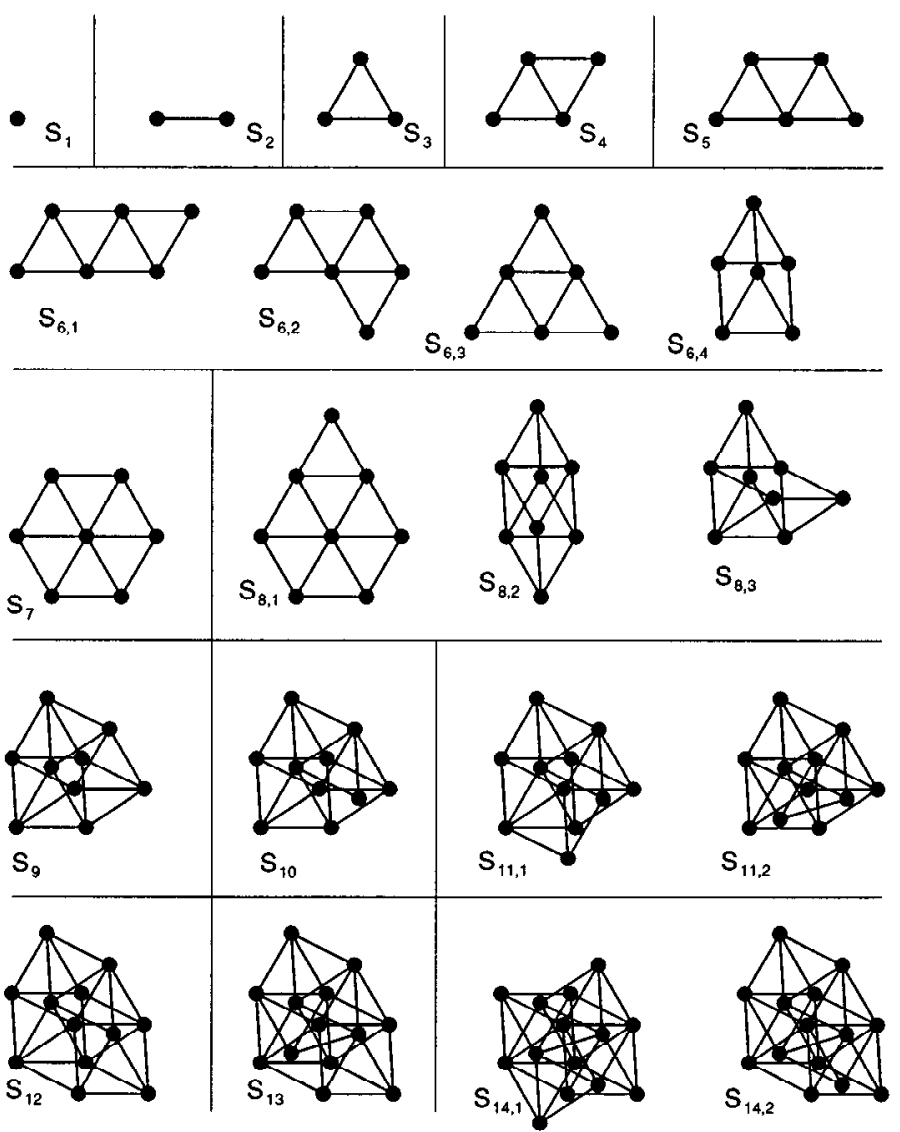

Fig. 1

were discussed, e.g., the maximum number of unit distances in other metric spaces, higher dimensions, convex or general positions, of smallest or largest distances, etc. (see [3] for further references).

The original problem, however, seems to have exhausted the available methods with the $O\left(n^{4 / 3}\right)$-bound. All known proofs of this upper bound used as main geometric information the fact that any two unit circles intersect in at most two points; and this information is used best possible in that bound, since there are strictly convex norms on $\mathbb{R}^{2}$ (which have the same intersection pattern) for which $c n^{4 / 3}$ unit distances among $n$ points are possible [3]. Attempts to enumerate the small excluded substructures of unit distance graphs [8], [14] did not yield any other excluded substructure that could be used to reduce this upper bound. Also the Schade sets do not support the implicit belief that lattice sections are extremal sets, since the larger sets are not subsets of any lattice [3], although the rational dimension is still small (they are generated by four unit vectors).

In the following all points will be in the plane, and we will distinguish pointsets only up to isometry. 


\section{The Result}

In the following we try to avoid these difficulties by proving structural results for the extremal sets. Since a lattice subset structure in itself is rather useless (for it may be a very thin subset), we aim for a lattice section structure. We call a set $S \subset \Gamma$ a section of $\Gamma$ if there is a convex set $K$ such that all points of $\Gamma$ that are in the interior of $K$ belong to $S$, and all points of $\Gamma$ that are in the exterior of $K$ do not belong to $S$. We make no assumption on the lattice points on the boundary of $K$; this allows incremental construction of sections for all numbers of points in $S$.

To obtain results, we introduce an additional structure on the pointsets with many unit distances by counting only those unit distances that occur in a few fixed directions. Let $S \subset \mathbb{R}^{2}$ be a set of $n$ points and let $T \subset \mathbb{R}^{2}$ be a set of $k$ unit vectors with $T \cap(-T)=\emptyset$ (we count each direction only once), and let

$$
f(S, T):=|\{(x, y) \in S \times S \mid x-y \in T\}|
$$

be the number of unit distances in $S$ which occur in directions from $T$. We study now the function $f(n, k)$ defined as the maximum of $f(S, T)$ over all such sets $S$ of $n$ points and $T$ of $k$ unit vectors. Trivially $f(n, 1)=n-1$. The unit distance graphs with unit distances in two directions are subgraphs of the union of disjoint copies of the square lattice graph; the maximum edge-number of a subgraph of the square-lattice graph was determined by Harary and Harborth [10], so we have $f(n, 2)=\lfloor 2 n-2 \sqrt{n}\rfloor$. The maximum number of edges in the triangular-lattice graph follows from a disk-packing theorem by Harborth [11]; this suggests that $f(n, 3)=\lfloor 3 n-\sqrt{12 n-3}\rfloor$. We prove:

Theorem. For each fixed $k$ we have $f(n, k)=k n-\Theta(\sqrt{n})$, and for $k \geq 3$ there is $a$ finite number of nonisometric lattices $\Gamma_{k, 1}, \ldots, \Gamma_{k, l_{k}}$ such that for each sufficiently large $n>n_{0}(k)$ each extremal set pair $S_{n, k}, T_{n, k}$ is a pair of subsets of one of the $\Gamma_{k, i}$.

There is always an extremal set $S_{n, k}$ that is, with the exception of at most $O(\sqrt{n})$ points, a section of that $\Gamma_{k, i}$ which is bounded by edges parallel to the vectors from $T_{n, k}$.

So for a fixed number of directions and a large number of points the extremal sets have a lattice section structure, and they are "equilateral sections" (see Lemma 2) like the set in Fig. 2. If the numbers $n_{0}(k)$ were small, this would imply the Erdôs conjecture, since each extremal set for the unrestricted maximum number of unit distances must be an extremal set for some number of directions $k$; but the Schade sets show that we cannot do without the lower bounds $n_{0}(k)$.

\section{The Proof}

In the following we denote for any pointset $X$ the unit distance graph of $X \subset \mathbb{R}^{2}$ by $G(X)$, and if additionally a set $Y \subset \mathbb{R}^{2}$ of vectors is given, and the graph of vertex pairs from $X$ with a difference in $Y$ by $G_{Y}(X)$. The edge-number of graph $G$ is written as $e(G)$.

The lower bound $f(n, k) \geq k n-O(\sqrt{n})$ is simple: Just select some $r$ such that the unit-distance graph $G\left(r \mathbf{Z}^{2}\right)$ is regular of degree at least $2 k$, e.g., $r=5^{-(1 / 2)\lceil k / 4\rceil}$. 


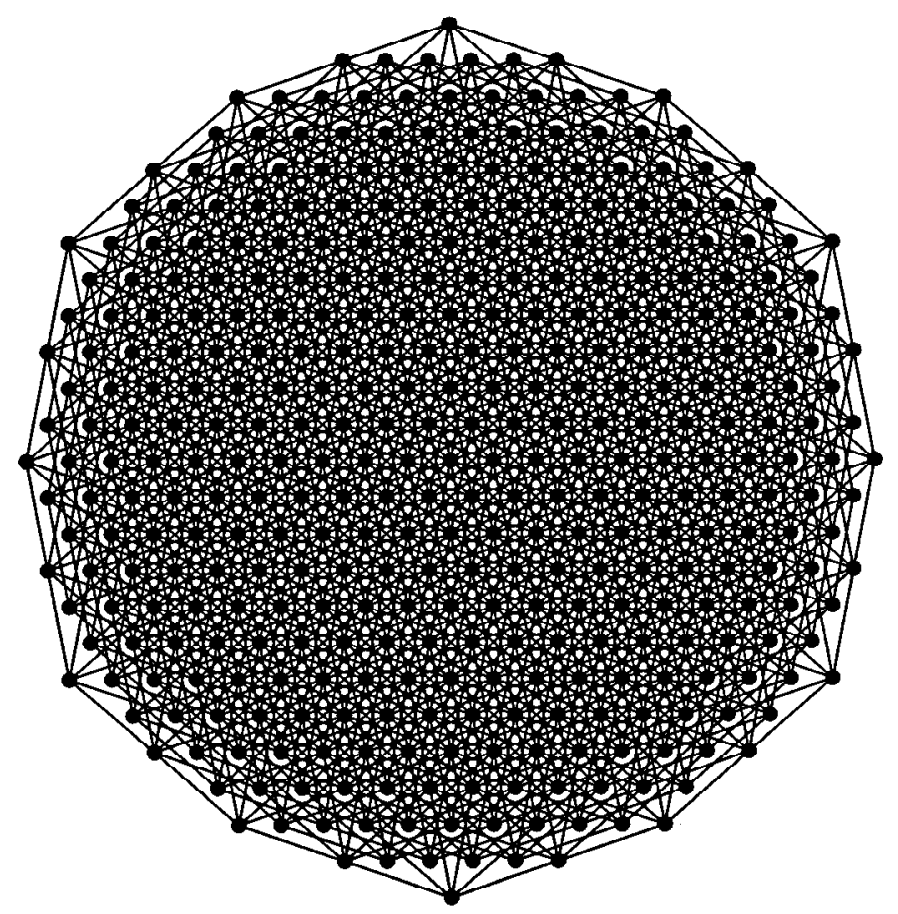

Fig. 2

Then we can select a set of $k$ unitvectors $T \subset r \mathbf{Z}^{2}$ (with $T \cap(-T)=\emptyset$ ) and a set of $n$ points $S:=r\left(\mathbf{Z}^{2} \cap[1, \sqrt{n}]^{2}\right)$. In this set $S$, each point with distance at least 1 to the boundary of the section (each point in $[r+1, r \sqrt{n}-1]^{2}$ ) has a neighbor in $S$ in each direction of $T$, so each of these points has degree $2 k$ in $G_{T}(S)$. Therefore $f(n, k) \geq e\left(G_{T}(S)\right)>k(n-(4 / r) \sqrt{n})$.

The theorem is now obtained from the following two lemmas:

Lemma 1. For each $k \geq 3$ there is a finite number of nonisometric lattices $\Gamma_{k, 1}, \ldots, \Gamma_{k, l_{k}}$ such that for all $n>n_{0}(k)$ each extremal pair $\left(S_{n, k}, T_{n, k}\right)$ is isometric to a pair of subsets of one of the $\Gamma_{k, i}$.

Lemma 2. Let $\Gamma \subset \mathbb{R}^{2}$ be a lattice and let $T \subset \Gamma \backslash\{0\}$ be a set of vectors such that the generated graph $G_{T}(\Gamma)$ is connected, and $T \cup(-T)$ does not contain three collinear vectors. Let $k:=\frac{1}{2}|T \cup(-T)|$.

Let $f(n)$ denote the maximum number of edges of a subgraph of $G_{T}(\Gamma)$ with $n$ vertices, and let $h(n)$ denote the maximum edge-number in an n-vertex subgraph that is generated by a section of $\Gamma$. Then for $n \geq n_{0}(T, \Gamma)$ the extremal sections for $h(n)$ are sections bounded by edges parallel to the graph-edges which are of relative (graph-) lengths that differ by at most one, and the extremal subsets for $f(n)$ differ from the extremal sections by at most $O(\sqrt{n})$ points. Also $f(n)=h(n)+O(1)=k n-c_{T, \Gamma} \sqrt{n}+\Theta(1)$. 
The assumption of no collinear generating vectors is necessary for the "almost equilateral structure" of the extremal sections, as can be seen by the graph generated on $\mathbf{Z}^{2}$ by the vectors $\{(1,0),(0,1),(0,2)\}$. We conjecture that for $n \geq n_{0}(T, \Gamma)$ the extremal subsets are sections, so $f(n)=h(n)$.

Proof of Lemma 1. Let $(S, T)$ denote an extremal pair for $f(n, k)$. By translation invariance we may assume $0 \in S$. If $G_{T}(S)$ were not connected, we could move one of the connected components against the others until a new unit vector of $T$ occurs; but then the set was not extremal. So for extremal $S$ the graph $G_{T}(S)$ is connected. Therefore each point of $S$ can be reached from the point 0 by following the $T$-edges; thus each point of $S$ is an integer linear combination of $T$-vectors: $S \subset\langle T\rangle_{Z}$.

Let $T=\left\{t_{1}, \ldots, t_{k}\right\}$, so $G_{T}(S)$ has maximum degree $2 k$. For each decomposition $T=T_{1} \cup \cdots \cup T_{l}$ into disjoint subsets we have

$$
k n-e\left(G_{T}(S)\right)=\sum_{i=1}^{l}\left(\left|T_{i}\right| n-e\left(G_{T_{i}}(S)\right)\right),
$$

and each summand is positive, $e\left(G_{T_{i}}(S)\right) \leq f\left(|S|,\left|T_{i}\right|\right)<\left|T_{i}\right||S|$. Since each $t_{i}$ is a unit vector, and $T \cap(-T)=\emptyset$, any two distinct $t_{i} \neq t_{j}$ are rationally independent. Therefore each graph $G_{\left\{t_{i}, t_{j}\right\}}(S)$ is an $n=|S|$-vertex subgraph of the classical square lattice graph. $G\left(\mathbf{Z}^{2}\right)=P_{\infty} \times P_{\infty}$. By the theorem of Harary and Harborth [10] we have $e\left(G_{\left\{t_{i}, t_{j}\right\}}(S)\right) \leq\lfloor 2 n-2 \sqrt{n}\rfloor$. Taking the sum over all 2-element subsets of $T$ we find

$$
e\left(G_{T}(S)\right) \leq k n-k \sqrt{n} .
$$

This proves $f(n, k)=k n-\Theta(\sqrt{n})$.

Suppose now that $T$ contains three rationally independent unit vectors $t_{1}, t_{2}, t_{3}$. Then the graph $G_{\left\{t_{1}, t_{2}, t_{3}\right\}}(S)$ is isomorphic to a subgraph of the unit distance graph $G\left(\mathbf{Z}^{3}\right)$ of the three-dimensional integer lattice. For we can assign each point of $S$ in each connected component of $G_{\left\{t_{1}, t_{2}, t_{3}\right\}}(S)$ integer coordinates relative to the basis $t_{1}, t_{2}, t_{3}$ and an arbitrary starting point of that component, with the edges corresponding to the point pairs with only one coordinate differing by exactly one. But an $n$-vertex subgraph of the three-dimensional lattice graph $G\left(\mathbf{Z}^{3}\right)$ contains less than $3 n-\left(\frac{9}{2} \pi\right)^{1 / 3} n^{2 / 3}$ edges (the exact maximum number is not known; probably cubic sections are optimal. An upper bound on the number $e$ of unit distances in a set $X \subset \mathbf{Z}^{3}$ of $n$ points is easily obtained by considering the Minkowski sum $Y:=X+\left[-1 / 2, \frac{1}{2}\right]^{3}$. If $A$ is the surface area of $Y$, then $3 n-\frac{1}{2} A \geq e$. The volume of $Y$ is at least $n$, so by the isoperimetric theorem we have $A \geq\left(36 \pi n^{2}\right)^{1 / 3}$, and thus $e \leq 3 n-\left(\frac{9}{2} \pi\right)^{1 / 3} n^{2 / 3}$.). So if for an extremal pair $(S, T)$ the set $T$ contains three rationally independent vectors, then $f(n, k) \leq k n-\left(\frac{9}{2} \pi\right)^{1 / 3} n^{2 / 3}$; this is for $n>n_{0}(k)$ a contradiction to our lower bound $f(n, k)>k n-c_{k} \sqrt{n}$. Therefore the set of integer combinations $\langle T\rangle_{Z}$ is for fixed $k$ and sufficiently large $n$ always a lattice (rational dimension 2), and $S$ is a subset of this lattice.

Next we will show that for each fixed $k \geq 3$ there are only finitely many nonisometric lattices that can occur as the underlying lattice $\Gamma:=\langle T\rangle_{Z}$ of the extremal sets $(S, T)$ (which we again assume to be an extremal pair for $f(n, k)$ ). $\Gamma$ is the thinnest lattice that contains $S$. Now let $t_{a}, t_{b} \in T$ be two distinct directions from $T$, then $2 n-2 \sqrt{n} \geq$ 
$e\left(G_{\left\{t_{a}, t_{b}\right\}}(S)\right) \geq 2 n-c_{k} \sqrt{n}$, since the defect in two directions is smaller than the total defect $c_{k} \sqrt{n}$. The vectors $t_{a}, t_{b}$ generate a sublattice $\left\langle t_{a}, t_{b}\right\rangle_{Z} \subset \Gamma$, and $\Gamma /\left\langle t_{a}, t_{b}\right\rangle_{Z}$ consists of a finite number $r=r\left(t_{a}, t_{b}\right)$ of cosets $\Gamma_{1}, \ldots, \Gamma_{r}$, which are translates of the sublattice $\left\langle t_{a}, t_{b}\right\rangle_{Z}$. The remaining unit vectors $t_{i}(i \neq a, b)$ of $T$ operate on these cosets, since they are lattice vectors in $\Gamma$; so for each $\Gamma_{j}, t_{i}$ the sum set $\Gamma_{j}+t_{i}$ is another of those finitely many cosets. But at least $\left|S \cap \Gamma_{j}\right|-\left|S \cap\left(\Gamma_{j}+t_{i}\right)\right|$ of the points of $S \cap \Gamma_{j}$ have no neighbor in $S$ in the direction $t_{i}$. Taking the sum over all cosets $\Gamma_{j}$ and all directions $t_{i}$ we find

$$
\sum_{j=1}^{r} \sum_{i=1}^{k}|| S \cap \Gamma_{j}|-| S \cap\left(\Gamma_{j}+t_{i}\right)|| \leq k n-f(n, k) \leq c_{k} \sqrt{n} .
$$

Since $T$ generates $\Gamma$, the graph generated by the $t_{i}$ on the cosets is connected; so we get from the previous inequality, especially,

$$
\max _{i=1}^{r}\left|S \cap \Gamma_{i}\right|-\min _{i=1}^{r}\left|S \cap \Gamma_{i}\right| \leq c_{k} \sqrt{n} .
$$

Now let $n_{i}:=\left|S \cap \Gamma_{i}\right|$. Then the graph $G_{\left\{t_{a}, t_{b}\right\}}\left(S \cap \Gamma_{i}\right)$ contains between the $n_{i}$ vertices at most $\left\lfloor 2 n_{i}-2 \sqrt{n_{i}}\right\rfloor$ edges. Since $G_{\left\{t_{a}, t_{b}\right\}}(S)$ contains at least $2 n-c_{k} \sqrt{n}$ edges, we have

$$
\sum_{i=1}^{r} \sqrt{n_{i}} \leq \frac{c_{k}}{2} \sqrt{n}
$$

We now look for the minimum of $\sum_{i=1}^{r} \sqrt{n_{i}}$ under the restrictions $\sum_{i=1}^{r} n_{i}=n$, $n_{i} \geq 0$, for $i=1, \ldots, r$ and $\max _{i=1}^{r} n_{i}-\min _{i=1}^{r} n_{i} \leq c_{k} \sqrt{n}$. Since $\sqrt{x}$ is a concave function, for the extremal choice of the $n_{i}$ each variable (with at most one exception) will meet a restriction of the admissible set. So among the $n_{i}$ there are only two distinct values,

$$
\max _{i=1}^{r} n_{i}=: \frac{n}{r}+\alpha c_{k} \sqrt{n} \quad \text { and } \quad \min _{i=1}^{r} n_{i}=: \frac{n}{r}+(\alpha-1) c_{k} \sqrt{n},
$$

where the last has to be nonnegative.

If $\min _{i=1}^{r} n_{i} \geq n / 2 r$, then we have

$$
\sum_{i=1}^{r} \sqrt{n_{i}}>r \sqrt{\min _{i=1}^{r} n_{i}} \geq r \sqrt{\frac{n}{2 r}}=\sqrt{\frac{r}{2}} \sqrt{n} .
$$

By $(*)$ we find that in this case $r$ is bounded by a constant for fixed $k$ and sufficiently large $n$.

If $0 \leq \min _{i=1}^{r} n_{i}<n / 2 r$, that is, $0 \leq n / r+(\alpha-1) c_{k} \sqrt{n}<n / 2 r$, we obtain $\max _{i=1}^{r} n_{i}=\min _{i=1}^{r} n_{i}+c_{k} \sqrt{n} \geq c_{k} \sqrt{n}$, and $(1-\alpha) \geq \sqrt{n} / 2 c_{k} r$. Since $\sum_{i=1}^{r} n_{i}=n$ holds, the smaller value of the $n_{i}$ must occur $\alpha r$ times and the larger value $(1-\alpha) r$ times. Therefore we have

$$
\sum_{i=1}^{r} \sqrt{n_{i}}=(1-\alpha) r \sqrt{\max _{i=1}^{r} n_{i}}+\alpha r \sqrt{\min _{i=1}^{r} n_{i}}
$$




$$
\begin{aligned}
& \geq(1-\alpha) r \sqrt{c_{k} \sqrt{n}} \geq \frac{\sqrt{n}}{2 c_{k} r} r \sqrt{c_{k} \sqrt{n}} \\
& \geq \frac{1}{2 \sqrt{c_{k}}} n^{3 / 4} .
\end{aligned}
$$

By $(*)$ we find that this case cannot occur for large $n$.

So for fixed $k$ and sufficiently large $n$ there are only finitely many possible values for $r(\Gamma, T)=\max r\left(t_{a}, t_{b}\right)$. To show now that for bounded $r(\Gamma, T)$ and $k \geq 3$ there are only finitely many nonisometric lattices $\Gamma$ possible, we look at the sublattices of $\Gamma$ generated by the three vectors $t_{i_{1}}, t_{i_{2}}, t_{i_{3}}$. If all these sublattices are known (up to isometry), then $\Gamma$ is determined (up to isometry), since $\Gamma$ is generated by $T$. Since $t_{i_{1}}, t_{i_{2}}, t_{i_{3}}$ are vectors of a lattice, they are rationally dependent, so there are relatively prime integers $a_{1}, a_{2}, a_{3}$ such that $a_{1} t_{i_{1}}+a_{2} t_{i_{2}}+a_{3} t_{i_{3}}=0$. These integers $a_{i}$ are nonzero, since $T \cap(-T)=\emptyset$, $t_{i} \neq \pm t_{j}$ for $i \neq j$. By the coefficients $a_{1}, a_{2}, a_{3}$ and the fact that all $t_{i}$ are unit vectors, the lattice $\left\langle t_{i_{1}}, t_{i_{2}}, t_{i_{3}}\right\rangle_{Z}$ is determined up to isometry, since $\left\|t_{i_{1}}\right\|=1,\left\|t_{i_{2}}\right\|=1$, and $\cos \left(\angle t_{i_{1}}, t_{i_{2}}\right)=\left(t_{i_{1}}, t_{i_{2}}\right)=\left(a_{3}^{2}-a_{2}^{2}-a_{1}^{2}\right) / 2 a_{1} a_{2}$. We now take the sublattice generated by the two vectors $t_{i_{1}}$ and $t_{i_{2}}$ of the lattice generated by all three vectors $\left\langle t_{i_{1}}, t_{i_{2}}, t_{i_{3}}\right\rangle_{Z}$, which itself is a sublattice of $\Gamma$. The number of cosets of $\left\langle t_{i_{1}}, t_{i_{2}}\right\rangle_{Z}$ in $\Gamma$ is at least as large as the number of cosets of $\left\langle t_{i_{1}}, t_{i_{2}}\right\rangle_{Z}$ in $\left\langle t_{i_{1}}, t_{i_{2}}, t_{i_{3}}\right\rangle_{Z}$; but that is $\left|a_{3}\right|$. Therefore the coefficients $a_{1}, a_{2}, a_{3}$ are bounded in absolute value by $r$. So for each sublattice of $\Gamma$ generated by three unit vectors there are at most $(2 r)^{3}$ nonisometric possibilities. So for fixed $k$ and sufficiently large $n$ there are only finitely many nonisometric lattices $\Gamma$ possible. This proves Lemma 1.

Proof of Lemma 2. Let $S_{n}$ and $X_{n}$ denote the extremal subset and section of $n$ points, respectively. Each point of an extremal set $\left(X_{n+1}\right.$ or $\left.S_{n+1}\right)$ that is a vertex of the convex hull of that set has degree at most $k$ in the graph, since all its $T \cup(-T)$-neighbors are on one side of a supporting line through that point. Removing that point gives $f(n+1) \leq f(n)+k$ and $h(n+1) \leq h(n)+k$ for all $n$. We are therefore most interested in the numbers $n$ for which $f$ or $h$ grow by at most $k-1$.

In the first step we show that in the extremal sets $\left(X_{n}\right.$ or $\left.S_{n}\right)$ most points have a big neighborhood in which no point of $\Gamma$ is missing in the extremal set.

A square section of $\Gamma$ gives a lower bound $f(n) \geq h(n) \geq k n-O(\sqrt{n})$. Therefore in $S_{n}$ as well as in $X_{n}$ all points with $O(\sqrt{n})$ exceptions have full degree $2 k$, i.e., if $p$ belongs to the extremal set, then also $\left\{p \pm t_{i} \mid i=1, \ldots, k\right\}$. For each fixed distance $d_{G}$ in the graph, the $O(\sqrt{n})$ points that do not have full degree have only $O(\sqrt{n})$ neighbors with graph-distance (in $\left.G_{T}(\Gamma)\right)$ at most $d_{G}$; so for each fixed $r_{G}$ all but $O(\sqrt{n})$ points in the extremal sets are centers of balls of radius $r_{G}$ (in the graph-metric of $G_{T}(\Gamma)$ ) that completely belong to the extremal sets. For each Euclidean ball with Euclidean radius $r_{E}$ around a point of $\Gamma$ there is a number $r_{G}$ such that the ball of radius $r_{G}$ in the graph-metric contains the Euclidean ball of radius $r_{E}$. Therefore the same statement also holds for the Euclidean metric: for each fixed Euclidean distance $d_{E}$ all but $O(\sqrt{n})$ points of the extremal set have distance at least $d_{E}$ to the nearest lattice point outside the extremal set.

In the next step we show that in each extremal set most points are contained in sections that are bounded by edges parallel to the vectors of $T$. 
Let $P$ denote the open 0 -symmetric convex $2 k$-gon, whose edgevectors are the vectors of $T \cup(-T)$ and let $Z$ be the union of all those translates of $P$ that do not contain a lattice point from outside the extremal set $\left(\Gamma \backslash X_{n}\right.$ or $\Gamma \backslash S_{n}$, respectively). Since $P$ has finite Euclidean diameter, all but $O(\sqrt{n})$ points of the extremal set are centers of a translate of $P$ which is completely inside the extremal set, so $Z$ contains all but $O(\sqrt{n})$ points of the extremal set. $Z$ consists of finitely many connected components, each of which is an open polygon, bounded by edges parallel to the directions of $T$. We denote these connected components by $Z_{1}, \ldots, Z_{l}$. In the following we denote as the relative length of a segment with direction $t \in T$ the ratio of its length to that of $t$.

We claim that for those $n$ for which the function associated with the extremal set ( $h$ or $f$ ) grows by less than $k$, these connected components must indeed be convex polygons. For by the construction of $P$ we have the property that if $q$ is a point outside $P$ and $t \in T \cup(-T)$ is a vector such that the ray with direction $t$ starting in $q$ intersects the interior of $P$, then at least one of the points $q+t, q+2 t, q+3 t, \ldots$ is in the interior of $P$. This property carries over to any union of translates of $P$, so especially to each connected component of $Z+P$.

Suppose now that one of the connected components $Z_{i}$ is not convex, and increase the extremal set by all those points inside the smallest open convex polygon that contains $Z_{i}$ and is bounded by edges parallel to the directions of $T$. Let $v$ be the number of added vertices and $\eta$ the number of added edges; we claim $\eta \geq k v$, so $f(n+v) \geq f(n)+k v$ which contradicts the assumption on $n$. To count the number of additional edges we note that each edge $\left\{q, q+t_{i}\right\}$ belongs to exactly one infinite sequence $\left(q+a t_{i}\right)_{a=-\infty}^{\infty}$ (a one-dimensional sublattice), and each such sequence that intersects the enlarged polygon already intersects the original polygon $Z_{i}$. So if such a sequence contains new points, it already contained at least one old point of the extremal set, but the points of the enlarged set form an interval in that sequence. Then in that sequence there are at least as many new edges as new points. But each new point belongs to exactly $k$ such sequences, and each new edge to exactly 1 , so $\eta \geq k v$, which proves the claim.

Now we investigate the structure of the boundary of such a section component.

Since each $Z_{i}$ is convex and is the union of translates of $P$, each edge of $Z_{i}$ (which is the direction of some vector $t \in T$ ) contains at least one edge of the translate of $P$ touching that edge of $Z_{i}$, so it is at least as long as that vector $t$. We call this edge of $Z_{i}$ trivial if it is exactly as long as $t$, and nontrivial if it is longer. A nontrivial edge $\overline{a(a+\sigma t)}$ must contain lattice points $p$ from the complement of the extremal set in each part of relative length greater than 1 , since there are points that block movement of $P$ across that edge in each part of the edge. Since $p$ is a lattice point and $t$ is a lattice vector, there is at least one one-dimensional sublattice $\ldots p-t, p, p+t, \ldots$ of which a segment $\Sigma:=\{p+z t \mid z \in \mathbf{Z}\} \cap \overline{a(a+\sigma t)}$ of $\lfloor\sigma\rfloor$ or $\lfloor\sigma\rfloor+1$ points is contained in the edge (if $t$ is not a primitive lattice-vector, there will be several such sublattices). $\Sigma$ defines a path in $G_{T}(\Gamma)$, and each of the points of $\Sigma$ (with the possible exception of the endpoint $a$ and $a+\sigma t$ of the segment) has already $k-1$ neighbors in the extremal set. So if there is a point of $\Sigma$ already belonging to the extremal set, and it has a neighbor in $\Sigma$ (that is not one of the endpoints of the segment), then this neighbor could be added to the extremal set giving $k$ new edges. So for those $n$ in which the associated function ( $f$ or $h$ ) grows only by $k-1$, each such $\Sigma$ must either completely belong to the extremal set (possibly excepting the endpoints), or completely belong to its complement; and each nontrivial 
edge of a $Z_{i}$ will contain at least one such $\Sigma$ that completely belongs to the complement of the extremal set (since there are missing points on the edge that stop movement across it).

We also note that the existence of nontrivial edges implies the possibility to add a point of degree at least $k-1$; and in an extremal set there have to be nontrivial edges. For a connected component with only trivial edges contains only $O(1)$ points, among them several that are not of full degree $2 k$ in the graph of the extremal set. But there are at most $O(\sqrt{n})$ such points, so not all $n$ points of the extremal set can be contained in connected components with only trivial edges. So for all $n \geq n_{0}(T, \Gamma)$ we have $k \geq f(n+1)-f(n) \geq k-1$ and $k \geq h(n+1)-h(n) \geq k-1$.

With this information we can now determine the structure of the extremal sections $X_{n}$ and thus prove the first statement of the lemma. Since the extremal sections are generated by convex sets, they can contain only one connected component $Z$. If there are points of $X_{n}$ in the exterior of this component, then any edge of the component that separates these points from $Z$ must have relative length at most 2, for otherwise the convexity of the generating set would force a segment of relative length 1 of the edge to contain only points of $X_{n}$, contradicting the existence of points of $\Gamma \backslash X_{n}$ in every edge-segment of relative length 1 . But there are only $O(1)$ points beyond such a short edge possible, since they must lie in the triangle bounded by the lines through the previous and following edges. So we can remove these $l_{1}$ points beyond the short edge (losing at most $k$ edges per points, since we can remove them in the order in which they become available as vertices of the convex hull) as well as the $l_{2}$ points on the short edge (losing at most $k l_{2}-1$ edges, since they form a union of paths, each point with $k-1$ neighbors on the other side of the short edge), with $l_{1}+l_{2}=O(1)$. But since $Z$ contains all but $O(\sqrt{n})$ points of $X_{n}$, there must be a side of length $\Omega(\sqrt{n})$, along which we can select a missing onedimensional sublattice $\Sigma$ as described above. This $\Sigma$ determines a path of length $\Omega(\sqrt{n})$ in $G_{T}\left(\Gamma \backslash X_{n}\right)$, of which we can add a subpath of length $l_{1}+l_{2}$ to the set $X_{n}$ (replacing the points on and beyond the short-edge we removed). This gives again $k\left(l_{1}+l_{2}\right)-1$ new edges, it therefore does not decrease the edge-number, but the new graph allows extension by further points, each giving $k$ new edges (extending the subpath of $\Sigma$ ). So for those $n>n_{0}(T, \Gamma)$ with $h(n+1)=h(n)+(k-1)$ this cannot happen. For those $n$ the extremal sections are generated by a polygon with $T$-parallel edges, and for the other $n$ in between we can obtain extremal sections by removing points along an edge.

For those $n$ with $h(n+1)=h(n)+(k-1)$ the edges of the extremal sets must have almost equal relative lengths: it cannot happen that we remove a path of $l$ vertices along one edge of $Z$ (losing $k l-1$ graph-edges) and find another edge of $Z$ on which there is a missing path $\Sigma$ of length $l^{*} \geq l+1$ (in which case we could add $l+1$ points with $k(l+1)-1$ new edges, contradicting the assumption on $h(n+1))$.

So the extremal sections $X_{n}$ are generated by polygons with $T$-parallel edges of almost equal relative length (as a multiple of the corresponding vector of $T$ ). So each edge has a length of $\alpha_{t} \sqrt{n}+\Theta(1)$. To count the number of edges in $X_{n}$, we note that outside the neighborhoods of the $2 k$ vertices of the generating polygon, we lose edges only along the polygon-edges, at a rate proportional to the length of the edge (the constant of course also depending on the direction). This gives the asymptotics claimed in the lemma

$$
h(n)=k n-c_{T, \Gamma} \sqrt{n}+\Theta(1) .
$$

We also note that if we have a set consisting of a section of $n \geq n_{0}(T, \Gamma)$ vertices and 
any number of arbitrary further vertices, we can extend this set by any given number $m$ of vertices in such a way that we gain at least $h(n+m)-h(n)-1$ additional edges. To prove this, we extend at the same time the section in the given set and the optimal section and compare the numbers of edges. The points are added in several stages using $m_{0}+m_{1}+\cdots+m_{r}=m$ points, each going until either there are no further points left or we have reached a number of points $n+m_{0}+\cdots+m_{i}$ for which $h$ grows only by $k-1$ (so a path along the boundary of the extremal section has been completed). We first disregard the further points outside the given section, and count only those edges going from the added points to the section. Let $m_{0}=\min \{j \geq 0 \mid h(n+j+1)<h(n+j)+k\}$ and $m_{i+1}=\min \left\{j \geq 1 \mid h\left(n+m_{0}+\cdots+m_{i}+j+1\right)<h\left(n+m_{0}+\cdots+m_{i}+j\right)+k\right\}$. The extremal section of a given number of points minimizes the maximum relative length of its boundary edges, so the section of the given set always has an edge of at least the length of the edge of the extremal set. This edge may already contain some points of the section, after filling it up we can start a new edge, so we can always add the $m_{i}$ points in such a way that we gain at least $k m_{i}-1$ new edges. For all $i>0$ we have by definition $h\left(n+m_{0}+\cdots+m_{i}\right)-h\left(n+m_{0}+\cdots+m_{i-1}\right)=k m_{i}-1$. Only $h\left(n+m_{0}\right)-h(n)=k m_{0}$. This proves the claim, if there are no further points. For each further point we have met in our construction, we have to add a point to the final section with as many edges going to the section as we counted for the point when we met it. We met these points as parts of edge-paths being filled up, and since the edges of the final section are at least as long as the edge-length when we met them, we can just add such paths again, which proves the claim.

Now we have to determine the structure of the extremal subsets; of these we already know that they consist of some $T$-parallel convex sections $Z_{1}, \ldots, Z_{l}$ and at most $O(\sqrt{n})$ further scattered points.

Let $n_{i}:=\left|\Gamma \cap Z_{i}\right|$ be the number of points of $S_{n}$ in $Z_{i}$. By the isoperimetric inequality we find that the boundary of $Z_{i}$ has length $\Omega\left(\sqrt{n_{i}}\right)$, so there are at least $\Omega\left(\sqrt{n_{i}}\right)$ points in $Z_{i} \cap S_{n}$ which do not have the full degree in $G_{T}\left(S_{n}\right)$. Since the total number of these points in $S_{n}$ is $O(\sqrt{n})$, we have $\sum_{i=1}^{l} \sqrt{n_{i}} \leq \alpha \sqrt{n}$. Using $\sum_{i} n_{i}=n-O(\sqrt{n})$ we find $\max _{i} n_{i} \geq \varepsilon n$; so there is a big connected component. Also we note that there are only $O(\sqrt{n})$ connected components, since each summand is at least 1.

All those points of $S_{n}$ that do not lie in any of the $Z_{i}$ will nonetheless be near one of the $Z_{i}$, the distance will remain bounded by a constant $\delta$. For each point of $S_{n}$ that does not belong to any of the $Z_{i}$ has a graph-distance at most $d$ to a point of $S_{n}$ that does not have full degree in $G_{T}\left(S_{n}\right)$. So if in the graph-metric of $G_{T}\left(S_{n}\right)$ there is a ball with a big radius $\rho$ around a point of $S_{n}$ that contains only points which do not belong to any $Z_{i}$ ( $\hat{n} \geq \rho$ such points), then the average degree in the interior of that ball is at most $2 k-\varepsilon(d)$, and the number of edges leaving the ball is at most $O(\sqrt{\hat{n}})$. It follows that $f(n-\hat{n}) \geq f(n)-(2 k-\varepsilon) \hat{n}-O(\sqrt{\hat{n}})$; for sufficiently large $\hat{n}$, i.e., sufficiently large radius $\rho$, this gives a contradiction to $f(n) \geq f(n-\hat{n})+f(\hat{n}) \geq f(n-\hat{n})+k \hat{n}-O(\sqrt{\hat{\hat{n}}})$. So the maximum distance $\rho$ of a point not belonging to any $\bar{Z}_{i}$ to the nearest point in a $Z_{i}$ stays bounded: $\rho=O(1)$.

Let $Y_{i} \subset \mathbb{R}^{2}$ denote the set of points with distance at most $\rho$ to $Z_{i}$. The union $\bigcup_{i=1}^{l} Y_{i}$ contains all points of $S_{n}$. Two sets $Y_{i}, Y_{j}$ can overlap, but for any set $Y_{i}$ the total area overlapped by other $Y_{j}$ is bounded by a constant. For if $Y_{i}$ is overlapped along an edge 
with direction $t_{a}$ by other sets $Y_{j}$ with a total length $\lambda$, then we can increase $S_{n}$ by all those points on parallels to that edge that lie between $Z_{i}$ and $Z_{j}$. These points can be partitioned as above in arithmetic progressions with a difference $t_{a}$ (one-dimensional sublattices), where the number of distinct such progressions that fits between $Z_{i}$ and $Z_{j}$ is bounded by the distance $O(\rho)=O(1)$. In each such sequence $p, p+t_{a}, p+2 t_{a}, \ldots$ the first point generates at least $k-1$ new edges, and all further points (till the end of $Z_{i}$ ) generate at least $k$ new edges. But as soon as these parallels reach $Z_{j}$, each point gives at least one further edge across to $Z_{j}$, which gives $\Omega(\lambda)$ further edges. So if we added a total of $\mu$ new points, we got at least $k \mu-O(\rho)+\Omega(\lambda)$ additional edges, which gives a contradiction to $f(n+\mu) \leq f(n)+k \mu$ unless $\lambda$ stays bounded by a constant.

If there is only one connected component, the claim of the lemma follows directly. So we assume there are several components $Z_{1}, Z_{2}, \ldots$, which are ordered with decreasing size $n_{i}$. Also let $m_{i}:=\left|\left(Y_{i} \backslash Z_{i}\right) \cap S_{n}\right|$. For each constant $\sigma$ the claim of the proof follows for sufficiently big $n>n_{0}(\sigma)$, if $n_{2}<\sigma$ holds, since there are only at most $O(\sqrt{n})$ components. So we can assume that $n_{2}$ gets arbitrarily big.

Suppose now that $m_{2} \leq \frac{1}{2} n_{2}$ holds. Each set $Y_{i}$ contains at most $h\left(n_{i}\right)+k m_{i}+O(1)$ edges: at most $h\left(n_{i}\right)$ within $Z_{i}$, at most $k m_{i}$ edges by shelling those points of $Y_{i} \backslash Z_{i}$ which are not overlapped by another $Y_{j}$, and at most $O(1)$ edges from those $O(1)$ points, in which $Y_{i}$ is overlapped by other $Y_{j}$.

If we now remove the $n_{2}+m_{2}$ points of $S_{n} \cap Y_{2}$, and extend $Z_{1}$ by this number of points we lose at most $h\left(n_{2}\right)+k m_{2}+O(1)$ edges in $Y_{2}$ and gain at least $h\left(n_{2}+m_{2}+n_{1}\right)-h\left(n_{1}\right)-1$ edges around $Z_{1}$. But using $m_{2} \leq \frac{1}{2} n_{2}$ we find

$$
\begin{aligned}
\left(h \left(n_{2}+m_{2}+\right.\right. & \left.\left.n_{1}\right)-h\left(n_{1}\right)-1\right)-\left(h\left(n_{2}\right)+k m_{2}+O(1)\right) \\
= & \left(k n_{2}+k m_{2}-c_{k}\left(\sqrt{n_{2}+m_{2}+n_{1}}-\sqrt{n_{1}}\right)+O(1)\right) \\
& -\left(k n_{2}+k m_{2}-c_{k} \sqrt{n_{2}}+O(1)\right) \\
= & -c_{k} \frac{n_{2}+m_{2}}{\sqrt{n_{1}+n_{2}+m_{2}}+\sqrt{n_{1}}}+c_{k} \sqrt{n_{2}}-O(1) \\
> & -c_{k} \frac{n_{2}+m_{2}}{2 \sqrt{n_{1}}}+c_{k} \sqrt{n_{2}}-O(1) \\
> & \frac{c_{k}}{4} \sqrt{n_{2}}-O(1),
\end{aligned}
$$

so for sufficiently big $n_{2}$ this increased the number of edges, a contradiction to the maximality of $S_{n}$.

And finally, if $m_{2}>\frac{1}{2} n_{2}$ holds and $n_{2}$ is sufficiently large, then the set $Z_{2}$ is not "round," but in one direction of length $\Omega\left(n_{2}\right)$, and orthogonal to that of width bounded by a constant. In that case a positive fraction of the points of $Z_{2}$ do not have full degree $2 k$; by removing the points of $Y_{2}$ we therefore lose at most $k m_{2}+(k-\varepsilon) n_{2}$ edges, and by increasing $Z_{1}$ we gain again, at least,

$$
k n_{2}+k m_{2}-c_{k}\left(\sqrt{n_{1}+n_{2}+m_{2}}-\sqrt{n_{1}}\right) \geq k n_{2}+k m_{2}-O\left(\sqrt{n_{2}}\right)
$$

edges $\left(m_{2}=O\left(n_{2}\right)\right)$, which again generates for sufficiently large $n_{2}$ a contradiction.

So all but $O(\sqrt{n})$ points of $S_{n}$ are in the first connected component, which was shown to be a convex polygonal section of $\Gamma$ bounded by edges parallel to $T$. Since we can 
remove the $m=O(\sqrt{n})$ outside points as they become available as vertices of the convex hull of the set, we have at most $h(n-m)+k m$ edges; since $\sqrt{n}-\sqrt{n-m}=O(1)$, we have $f(n)=h(n)+O(1)$. But this implies that the edge-lengths of that component can differ by at most $O(1)$ from the edge-lengths of the optimal section, so the optimal subset differs from the optimal section by at most $O(\sqrt{n})$ points.

This completes the proof of Lemma 2 and the theorem.

\section{References}

1. J. Beck and J. Spencer. Unit distances, J. Combin. Theory Ser. A, 37 (1984), 231-238.

2. P. Brass. Erdős distance problems in normed spaces, Comput. Geom. Theory Appl., 6 (1996), 195-214.

3. P. Brass. Extremale Konstruktionen in der kombinatorischen Geometrie, Habilitationsschrift, Universität Greifswald, 1996.

4. P. Brass. Häufige Abstände in endliche Punktmengen, Jahrbuch Überblicke Mathematik, 1998, pp. 66-75.

5. K. L. Clarkson, H. Edelsbrunner, L. J. Guibas, M. Sharir, and E. Welzl. Combinatorial complexity bounds for arrangements of curves and spheres, Discrete Comput. Geom., 5 (1990), 99-160.

6. K. B. Chilakamarri. Unit distance graphs in Minkowski metric spaces, Geom. Dedicata, 37 (1991), 345356.

7. K. B. Chilakamarri. The unit-distance graph problem: A brief survey and some new results, Bull. ICA, 8 (1993), 39-60.

8. K. B. Chilakamarri and C. R. Mahoney. Maximal and minimal forbidden unit-distance graphs in the plane, Bull. ICA, 13 (1995), 35-43.

9. P. Erdôs. On sets of distances of $n$ points, Amer. Math. Monthly, 53 (1946), 248-250.

10. F. Harary and H. Harborth. Extremal animals, J. Combin. Inform. System Sci., 1 (1976), 1-8.

11. H. Harborth. Lösung zu Problem 664A, Elem. Math., 29 (1974), 14-15.

12. S. Józsa and E. Szemerédi. The number of unit distances in the plane, In: Infinite and Finite Sets (A. Hajnal et al., eds.), North-Holland, Amsterdam, 1975, pp. 939-950.

13. J. Pach and P. K. Agarwal. Combin. Geom., Wiley, New York, 1995.

14. C. Purdy and G. Purdy. Minimal forbidden distance one graphs, Congr. Numer., 66 (1988), 165-172.

15. C. Schade. Exakte Maximalzahlen gleicher Abstände, Diplomarbeit bei H. Harborth, Universität Braunschweig, 1993.

16. J. Spencer, E. Szemerédi, and W. Trotter. Unit distances in the Euclidean plane, In: Graph Theory and Combinatorics (B. Bollobás, ed.), Academic Press, London, 1984, pp. 293-304.

17. L. A. Székely. Crossing numbers and hard Erdős problems in discrete geometry, Preprint, 1996.

Received January 10, 1997, and in revised form May 16, 1997. 